\title{
Negative band gap bowing in epitaxial InAs/GaAs alloys and predicted band offsets of the strained binaries and alloys on various substrates
}

\author{
Gus L. W. Hart \\ gus.hart@gmail.com \\ Kwiseon Kim
}

Alex Zunger

Follow this and additional works at: https://scholarsarchive.byu.edu/facpub

Part of the Astrophysics and Astronomy Commons, and the Physics Commons

\section{Original Publication Citation}

K. Kim, G. L. W. Hart, and A. Zunger, "Negative Band Gap Bowing in Epitaxial InAs/GaAs Alloys and Predicted Band Offsets of the Strained Binaries and Alloys on Various Substrates," Appl. Phys. Lett. 8315 (29 April 22). The original article may be found here: http://apl.aip.org/ resource/1/applab/v8/i17/p315_s1

\section{BYU ScholarsArchive Citation}

Hart, Gus L. W.; Kim, Kwiseon; and Zunger, Alex, "Negative band gap bowing in epitaxial InAs/GaAs alloys and predicted band offsets of the strained binaries and alloys on various substrates" (2002). Faculty Publications. 546.

https://scholarsarchive.byu.edu/facpub/546

This Peer-Reviewed Article is brought to you for free and open access by BYU ScholarsArchive. It has been accepted for inclusion in Faculty Publications by an authorized administrator of BYU ScholarsArchive. For more information, please contact ellen_amatangelo@byu.edu. 


\title{
Negative band gap bowing in epitaxial InAs/GaAs alloys and predicted band offsets of the strained binaries and alloys on various substrates
}

\author{
Kwiseon Kim, Gus L. W. Hart, ${ }^{\text {a) }}$ and Alex Zunger ${ }^{\text {b) }}$ \\ National Renewable Energy Laboratory, Golden, Colorado 80401
}

(Received 21 December 2001; accepted for publication 26 February 2002)

\begin{abstract}
We use pseudopotential theory to provide (1) the band offsets of strained GaAs and InAs on various substrates and (2) the energies $E_{v}(x)$ and $E_{c}(x)$ of the valence and conduction bands of $\operatorname{In}_{x} \mathrm{Ga}_{1-x} \mathrm{As}$ alloy, as a function of composition. Results are presented for both the bulk alloy and for the alloy strained on InP or GaAs. We predict that while $E_{c}(x)$ bows downward for relaxed bulk alloys, it bows upward for strained epitaxial alloys. The calculated alloy offsets are used to discuss electron and hole localization in this system. (C) 2002 American Institute of Physics.
\end{abstract}

[DOI: 10.1063/1.1470693]

InAs and GaAs are the building blocks of a diverse range of systems, ${ }^{1}$ including short-period superlattices $(\text { InAs })_{n} /(\mathrm{GaAs})_{m}$ of the binary constituents, bulk alloys $\mathrm{In}_{x} \mathrm{Ga}_{1-x} \mathrm{As}$, epitaxial alloys that are coherently grown on a substrate (InP or GaAs), alloy superlattices or quantum wells $\left(\mathrm{In}_{x} \mathrm{Ga}_{1-x} \mathrm{As}\right)_{p} /(\mathrm{InP})_{q}$, and GaAs-embedded InAs quantum dots. A central quantity that controls many of the optical and transport properties of such systems are the band-edge energies $E_{v}$ and $E_{c}$ of the valence $(v)$ and conduction $(c)$ states, as a function of composition, strain, and material dimensionality. Two things are known about these quantities. First, the relative band offset $E_{v}(\mathrm{InAs} / \mathrm{GaAs})$ and $E_{c}(\mathrm{InAs} / \mathrm{GaAs})$ between the end-point binary compounds can be obtained either from direct photoemission ${ }^{2}$ or transport ${ }^{3}$ measurements or can be calculated using accurate first-principles calculations (summarized for all III-V's and all II-VI's in Ref. 4). Second, the pressure dependence of the band gap $E_{g}=E_{c}$ $-E_{v}$ (summarized in Ref. 5 for all zincblende semiconductors) are also known. What is generally not known directly about these quantities is: (i) the relative offset $E_{v, c}\left(\operatorname{In}_{x} \mathrm{Ga}_{1-x} \mathrm{As} / \mathrm{In}_{y} \mathrm{Ga}_{1-y} \mathrm{As}\right)$ as a function of alloy composition, and, (ii) the absolute pressure, or strain dependence of the individual band-edge energies $E_{v}$, and separately $E_{c}$. But knowledge of these quantities is crucial because the relative alloy band-edge offset and their absolute pressure dependence decides the confinement of holes or electrons in heterostructures made of strained alloy wells and barriers.

Alloy offsets, e.g., the offset between $\operatorname{In}_{x} \mathrm{Ga}_{1-x} \mathrm{As}$ and InP or GaAs, are generally unknown via direct measurements and are usually treated as an adjustable parameter (along with other quantities) when measured interband transition energies are fit to simple, effective-mass based models. ${ }^{6-8}$ Such fits produce a disappointingly large range of alloy band offsets. It is generally unknown how much of the uncertainty results from fundamental limitations in the underlying (effective-mass) physical model used in the fit and how much should be attributed to interdependence between the fitting parameters of the model (e.g., uncertainties in the strain-modified effective masses lead to uncertainties in the

\footnotetext{
a) Also At: Department of Physics and Astronomy, Northern Arizona University, Flagstaff, AZ 86011-6010; electronic mail: gus.hart@nau.edu

${ }^{b)}$ Electronic mail: azunger@nrel.gov
}

fitted band offsets). It is not uncommon to encounter equally precise optical measurements on InAs/GaAs systems, interpreted via widely different alloy band offsets. (E.g., the offset-partitioning ratio $Q_{c}=\Delta E_{c} / \Delta E_{g}$ can range ${ }^{6,9}$ from $30 \%$ to $70 \%$.) Theoretical studies that produce rather accurate offsets between binary constituents (e.g., Ref. 4 and references therein), are often too difficult to apply to alloy constituents, because large supercells are needed to capture the random alloy distribution.

Absolute pressure/strain dependencies of individual band edges are generally unknown because only the differences $E_{g}=E_{c}-E_{v}$ are measured. One can use the "vacuum pinning rule" 10 (which relates transition-atom defect levels to an absolute energy reference) to infer approximate bandedge pressure coefficients. ${ }^{11}$ Theoretical studies of absolute band-edge pressure coefficients ${ }^{12}$ are rather rare, as they require superlattice calculations for a given material in different strain configurations (e.g., a superlattice made of compressed and dilated GaAs). Recent theoretical results are summarized in Refs. 5 and 12 and predict that valence states have a different slope with pressure than previously accepted from a simpler, "spherical solid" atomic-like model. ${ }^{13}$ Specifically, instead of moving to more negative energies upon application of pressure, as suggested by atomic-like models, ${ }^{13}$ first-principles solid state calculations ${ }^{12}$ predict that, for GaP, GaAs, GaSb, InP, InAs, InSb, and for all IIVI's, $E_{v}$ moves to more positive energies. This new bandedge deformation potential was recently used successfully in many calculations of strained InAs/GaAs quantum dots. ${ }^{14}$ The purpose of the present letter is to derive a physical model of band-edge states in InAs/GaAs from electronic structure theory, rather than deduce it from fitting the experimental spectroscopy of the system. ${ }^{6-8}$

We describe here an approximate but accurate method that gives the variation in the band-edge energies $E_{v}$ and $E_{c}$ of InAs/GaAs systems as a function of alloy composition, strain, and atomic configuration. We give results for (a) InAs/ GaAs binaries on various substrates (GaAs, InP, and InAs), (b) $\mathrm{In}_{x} \mathrm{Ga}_{1-x} \mathrm{As} / \mathrm{In}_{y} \mathrm{Ga}_{1-y} \mathrm{As}$ alloy heterojunctions on various substrates. The band-edge energies of these systems are supplied graphically in Figs. 1-3 and can be used for future modeling of such systems.

We start by calculating the unstrained and strained band 


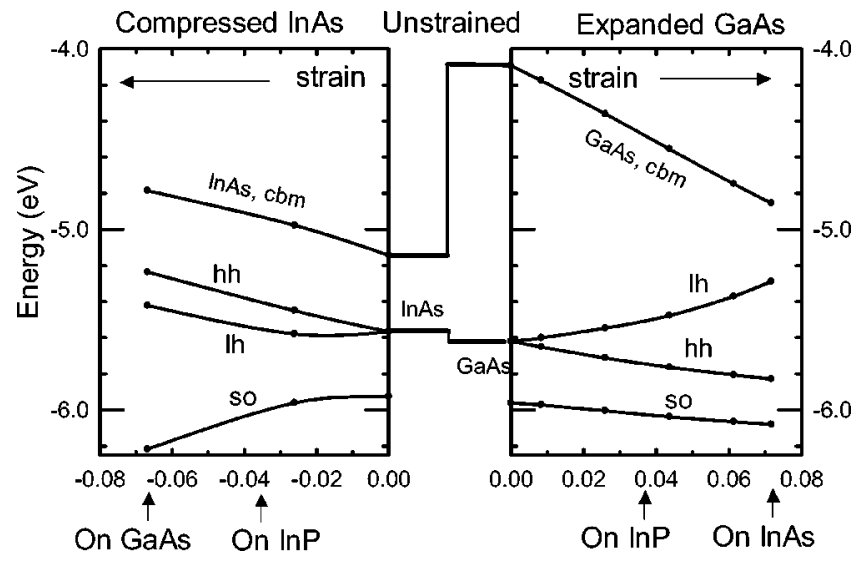

FIG. 1. LDA-calculated strain-modified eigenvalues of epitaxially expanded GaAs (on InP or InAs (001) substrates) and epitaxially compressed InAs (on InP or GaAs). The band gaps of the uncompressed solids are taken from experiment. The unstrained band offset is calculated in LDA (see Ref. 4). The energy zero refers to a vacuum.

offsets between the end-point binaries InAs/GaAs using the first-principles local density method, as implemented via the linear augmented plane wave (LAPW) approach. ${ }^{15}$ Reference 4 and references therein describe how one deduces from LAPW calculations the position of core levels of GaAs, InAs, and GaAs/InAs heterojunction relative to the respective band edges and how these quantities gives the band offsets. To first order, the calculated offset is unaffected by the "local density approximation (LDA) band gap error." Figure 1 shows our resulting band-edge energies for GaAs and InAs versus strain, where we combined our calculated LDA band offsets with the measured band gaps of the binaries. The band-edge energies are given as absolute energies with respect to the intrinsic vacuum level as zero. We see that the unstrained, "natural" valence offset (central panel of Fig. 1) is rather small $(52 \mathrm{meV})$ but it increases rapidly with strain. This is shown more clearly in Fig. 2 which depicts the offsets on three specific (001) substrates. On the GaAs substrate, the heavy-hole (hh) offset is $383 \mathrm{meV}$, the light-hole (lh) offset is $198 \mathrm{meV}$ (hole localized on InAs) and the conduction band offset is $693 \mathrm{meV}$. On the InAs substrate, we have a reversal of hole states, with $\mathrm{lh}$ above hh, giving a negative hh offset of $-261 \mathrm{meV}$, while the lh offset is 279 $\mathrm{meV}$ (hole localized on GaAs). The conduction band offset is now reduced to $289 \mathrm{meV}$. On the intermediate InP substrate, we have a hh offset of $316 \mathrm{meV}$, a lh offset of only 249-316 $=-76 \mathrm{meV}$ (hole localized on GaAs), and an intermediate conduction band offset of $465 \mathrm{meV}$. The calculated conduction band offset on GaAs substrate $(693 \mathrm{meV})$ compares well with the most recent experimental determination ${ }^{3}(690 \mathrm{meV})$

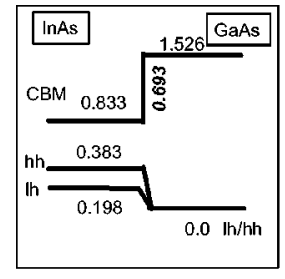

on GaAs

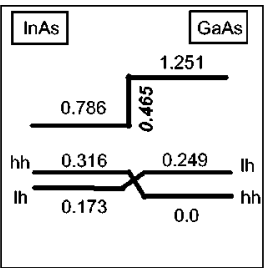

on $\ln P$

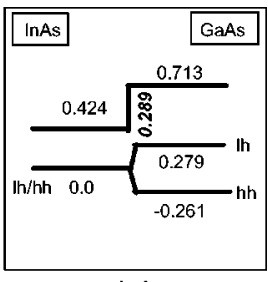

on $\ln A s$
FIG. 2. Calculated band offsets of InAs/GaAs on various (001) substrates. The horizontal numbers indicate the energy of each level (in eV). Differences of these numbers give the offsets.

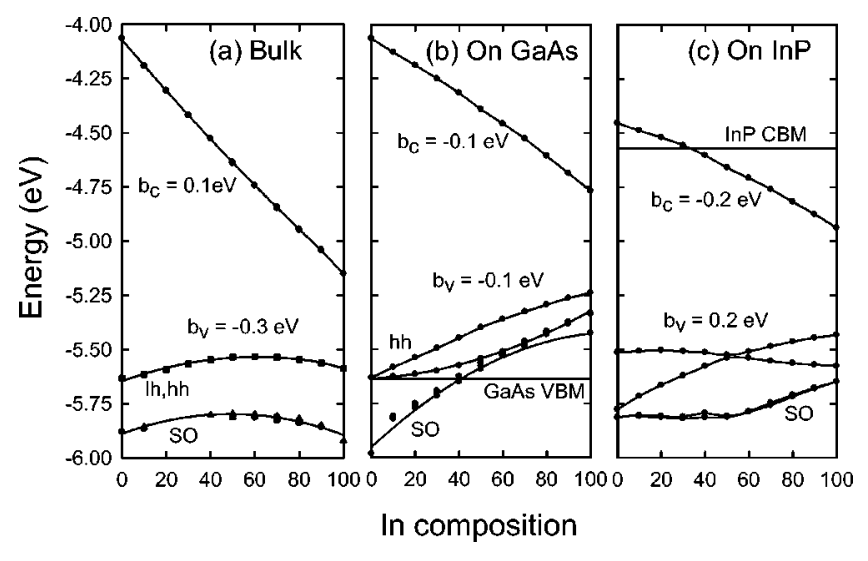

FIG. 3. Dependence of conduction and valence band-edge energies on alloy composition for (a) bulk alloy (i.e., relaxed alloy), (b) epitaxial alloy on GaAs (thin horizontal line denotes GaAs CBM), and (c) exitaxial alloy on InP (thin horizontal line denotes InP CBM).

but disagrees with the older experiment by Hirakawa et al. ${ }^{16}$ $(380 \mathrm{meV})$ which is, most likely, incorrect. The valence band offset $(383 \mathrm{meV})$ differs from the atomic-like estimate of Van de Walle ${ }^{13}(430 \mathrm{meV})$, the LDA value of Tit et al. ${ }^{17}(280$ $\mathrm{meV})$, or the photoemission value of Ohler et al. ${ }^{2}(-40$ $\mathrm{meV})$.

Having obtained from first principles the band-edge energies of the binary constituents, we now fit to them a more general, empirical pseudopotential ${ }^{14,18}$ for In, Ga, and As. This potential is fit to (i) the measured (not the LDA) band gaps of the binaries and their effective masses, (ii) the LDA calculated absolute deformation potentials ${ }^{5,12}$ and band offsets (Fig. 1), and (iii) the bowing of the bulk random alloy. Unlike the traditional "empirical pseudopotential method" 19 that upon examination was found to yield poor effective masses and deformation potentials, this new generation potential has an explicit dependence not only on momentum, but also on local strain ${ }^{14,18}$ and thus yields an accurate depiction of bands, masses, and strain effects. These new potentials have two advantages over the LDA approach: they do not have any "band gap error," and they can be expanded in a small basis set of plane waves (about 30 per atom, rather than hundreds per atom in LDA). Consequently, when coupled with "order N" Hamiltonian diagonalization methods ${ }^{20}$ they can be applied to supercells containing thousands of atoms, affording accurate descriptions of alloy fluctuation effects, ${ }^{21}$ superlattices $^{22}$ and quantum-dot structures. ${ }^{14}$

We predict the strain, composition, and configurational dependence of the alloy band-edge energies $E_{v}$ and $E_{c}$, as follows: We construct a "supercell" with $N$ cation and $N$ anion sites $\mathrm{In}_{N / 2} \mathrm{Ga}_{N / 2} \mathrm{As}_{N}$, placing the atoms on their respective face-centered-cubic sites. For a random alloy, sites are occupied randomly by In or Ga according to the composition. Once assigned to sites, atoms are allowed to displace locally, so as to minimize the strain energy as described via a valance force field. ${ }^{23}$ In "free-floating" bulk alloys, atoms are allowed to be displaced without constraint, whereas for strained epitaxial alloys, we fix the in-plane lattice constant and permit relaxation of the perpendicular axis, as well as of all cell-internal atomic positions, consistent with coherency on a given substrate. Once local equilibrium is achieved, we create the supercell pseudopotential by superposing the 
atomic pseudopotentials $v_{\alpha}$ of all atoms of type $\alpha$ at lattice position $R_{n \alpha}$. The Schroedinger equation

$$
\left\{-\frac{1}{2} \nabla^{2}+\sum_{n} \sum_{\alpha} \nu_{\alpha}\left(r-R_{n}-d_{\alpha}\right)\right\} \psi_{i}=\varepsilon_{i} \psi_{i}
$$

is then solved by expanding the wave function $\psi_{\mathrm{i}}$ in a plane wave basis, calculating all matrix elements numerically, essentially exactly, and using our "order $N$ " method ${ }^{20}$ to diagonalize the Hamiltonian matrix. Spin orbit is included (so the pseudopotential is nonlocal) and for random alloys, we repeat the process for a few, randomly created configurations, averaging the energies. For the alloy results discussed in this letter, we used 512-atom supercells and averaged over five different randomly generated configurations.

Figure 3 shows the calculated band-edge energies as a function of composition for the free-floating relaxed bulk alloy (part a), as well as for the strained (epitaxial) alloys or GaAs and InP substrates (parts b and c, respectively). We learn that:

(i) Epitaxy-induced reversal of bowing parameters. The bulk alloy (i.e., relaxed alloy) shows a downward-bowing conduction-band minimum (CBM) and an upward-bowing valence band minimum (VBM) (Fig. 3a), with a total band gap bowing coefficient $b_{\text {gap }}=b_{c}-b_{v}$ of $+0.4 \mathrm{eV}$, in good agreement with experiment ${ }^{24}$ and LDA calculations. ${ }^{25}$ The reason that $E_{v}$ bows upward with composition is that the highest valence states (anion $p$-like, $\Gamma_{8 v}$ ) is pushed up by the lower-lying, $\Gamma_{8 v}$-folding state $\mathrm{L}_{4,5 v}$ which is also anion $p$-like. ${ }^{26}$ On the other hand, $E_{c}$ bows downward with composition due to repulsion of the CBM (cation s-like $\Gamma_{6 c}$ ) by higher lying $\mathrm{L}_{6 c}$ and $\mathrm{X}_{6 c}$ cation s-like states. ${ }^{25,26}$ In contrast to the bulk alloys however, the strained epitaxial alloys (Figs. $3 \mathrm{~b}$ and $3 \mathrm{c}$ ) show an upward bowing CBM $\left(b_{c}<0\right)$, leading to a band gap bowing of $-0.1 \mathrm{eV}$ on GaAs and -0.2 $\mathrm{eV}$ on InP, much smaller than that of the bulk alloy. In In$\mathrm{GaN}$ alloys, epitaxy reduces the bowing coefficient from 4.1 to $3.4 \mathrm{eV}^{27}$ Clearly, in InGaN the $0.7 \mathrm{eV}$ reduction, although large, does not reverse the sign of the bowing. But in InGaAs, the epitaxial effect is sufficient to reverse the sign of the bowing. The reason for this reversal is strain effects: When the alloy is compressed (e.g., In-rich $\operatorname{In}_{x} \mathrm{Ga}_{1-x} \mathrm{As} / \mathrm{InP}$ ) its CBM moves up (much like in the zincblende constituents, see Fig. 1) relative to the unstrained alloy. For InAs/InP, this shift is $+195 \mathrm{meV}$. Conversely, when the alloy is expanded (e.g., Ga-rich $\operatorname{In}_{x} \mathrm{Ga}_{1-x} \mathrm{As} / \mathrm{InP}$ ) its CBM moves down. For $\mathrm{GaAs} / \mathrm{InP}$, this shift is $-390 \mathrm{meV}$. At the lattice-matched composition, $\operatorname{In}_{0.53} \mathrm{Ga}_{0.47} \mathrm{As} / \mathrm{InP}$, the $\mathrm{CBM}$ is unchanged. Since the upward displacement of the In-rich CBM segment is smaller than the downward displacement of the Ga-rich CBM segment, the CBM bowing changes sign via epitaxy. This epitaxy-induced reversal of bowing parameter effects the offset partitioning ratio $Q_{c}(x)=\Delta E_{c}(x) / \Delta E_{g}(x)$. That is, the conduction band to valence band offset ratio varies with composition. This effect was generally overlooked in fitting quantum-well spectra via adjustment of $Q$.

(ii) Hole localization: The thin horizontal line in Fig. 3(b) depicts the energy of the unstrained GaAs VBM. We see that the hh is higher in energy than the GaAs VBM and thus is localized on the alloy material, not on GaAs in the epitaxial alloy grown on GaAs [Fig. 3(b)]. However, at low In concentration, the $\mathrm{lh}$ in the InGaAs/GaAs system has a very low offset with GaAs, so the hole is expected to be delocalized. For $x_{\text {In }}>10 \%$, this hole is always localized on the Inrich material. For the epitaxial alloy on InP [Fig. 3(c)], we find a $\mathrm{lh}-\mathrm{hh} \operatorname{crossover}^{28}$ around $50 \%$ In, and a very shallow offset, suggesting rather delocalized holes.

(iii) Electron localization: In the bulk alloy [Fig. 3(a)] and in the epitaxial alloy on GaAs [Fig. 3(b)] the electrons are always localized on the In-rich material. The CBM of unstrained InP is shown as a dashed horizontal line in Fig. 3(c), exhibiting a crossover with the alloy CBM: for $x_{\text {In }}$ $<20 \%$, the electrons are localized on the InP substrate, whereas for $x_{\text {In }}>20 \%$ they are localized on the alloy. ${ }^{28}$

The results of Figs. 1-3 can be used directly in modeling in electronic transitions in alloy superlattices or quantum wells and are more accurate than effective-mass fits. They demonstrate (1) the change in sign of the band gap bowing coefficient as a relaxed, bulk alloy becomes coherent with a substrate, (2) the crossover of the CBM's of InP and GaInAs/ InP, (3) lh-hh crossover of InGaAs/InP versus composition, and (4) hole delocalization in InGaAs/GaAs.

This work was supported by US DOE, SC-BEC-DMS.

${ }^{1}$ C. R. Leavens and R. Taylor, "Interfaces, Quantum-wells and Superlattices," NATO ASI Series (Plenum, New York, 1987).

${ }^{2}$ C. Ohler, A. Forster, J. Mores, C. Daniels, and H. Luth, J. Phys. D 30, 1436 (1997).

${ }^{3}$ R. Colombelli, V. Piazza, A. Badolato, M. Lazzarino, F. Beltram, W. Schoenfeld, and P. Petroff, Appl. Phys. Lett. 76, 1146 (2000).

${ }^{4}$ S. H. Wei and A. Zunger, Appl. Phys. Lett. 72, 2011 (1998).

${ }^{5}$ S. H. Wei and A. Zunger, Phys. Rev. B 60, 5404 (1999).

${ }^{6}$ F. Ikawa, F. Cerdeira, C. Vazquez-Lopez, P. Motisuke, M. A. Sacilotti, A. P. Roth, and R. A. Masut, Phys. Rev. B 38, 8473 (1988).

${ }^{7}$ J.-Y. Marzin, M. N. Charasse, and B. Sermage, Phys. Rev. B 31, 8298 (1985).

${ }^{8}$ J. Menéndez, A. Pinczuk, D. J. Werder, S. K. Sputz, R. C. Miller, D. L. Sivco, and A. Y. Cho, Phys. Rev. B 36, 8165 (1987).

${ }^{9}$ B. Jogai and P. W. Yu, Phys. Rev. B 41, 12650 (1990).

${ }^{10}$ M. Caldas, A. Fazzio, and A. Zunger, Appl. Phys. Lett. 45, 671 (1984); A. Zunger, Phys. Rev. Lett. 54, 848 (1985).

${ }^{11}$ J. M. Langer and H. Heinrich, Phys. Rev. Lett. 55, 1414 (1985).

${ }^{12}$ A. Franceschetti, S. H. Wei, and A. Zunger, Phys. Rev. B 50, 17797 (1994).

${ }^{13}$ C. G. Van de Walle, Phys. Rev. B 39, 1871 (1989).

${ }^{14}$ L.-W. Wang, J. Kim, and A. Zunger, Phys. Rev. B 59, 5678 (1999).

${ }^{15}$ S.-H. Wei and H. Krakauer, Phys. Rev. Lett. 55, 1200 (1985), and references therein; D. J. Singh, Planewaves, Pseudopotential, and the LAPW Method (Kluwer, Boston, 1994), and references therein; P. Blaha, K. Schwarz, and J. Luitz, WIEN97, Vienna University of Technology, Vienna, 1997; P. Blaha, K. Schwarz, P. Sorantin, and S. B. Trickey, Comput. Phys. Commun. 59, 399 (1990).

${ }^{16}$ K. Hirakawa, Y. Hashimoto, K. Harada, and I. Ikoma, Phys. Rev. B 44, 1734 (1991).

${ }^{17}$ N. Tit, M. Peressi, and S. Baroni, Phys. Rev. B 48, 17607 (1993).

${ }^{18}$ A. Zunger, in Quantum Theory of Real Materials, edited by J. R. Chelikowsky and S. G. Louie (Kluwer, Boston 1996), p. 173; H. Fu and A. Zunger, Phys. Rev. B 55, 1642 (1997).

${ }^{19}$ M. L. Cohen and T. K. Bergstrasser, Phys. Rev. 141, 789 (1966).

${ }^{20}$ L. W. Wang and A. Zunger, J. Chem. Phys. 100, 2394 (1994).

${ }^{21}$ L. W. Wang, L. Bellaiche, S. H. Wei, and A. Zunger, Phys. Rev. Lett. 80, 4725 (1998).

${ }^{22}$ R. Magri and A. Zunger, Phys. Rev. B 62, 10364 (2000).

${ }^{23}$ P. Keating, Phys. Rev. 145, 637 (1966).

${ }^{24}$ S. Paul, J. B. Roy, and P. K. Basu, J. Appl. Phys. 69, 827 (1991); J. R. Jensen, J. M. Huam, and W. Langben, ibid., 86, 2584 (1999).

${ }^{25}$ S.-H. Wei and A. Zunger, Phys. Rev. Lett. 76, 664 (1996).

${ }^{26}$ S.-H. Wei and A. Zunger, Phys. Rev. B 39, 3279 (1989).

${ }^{27}$ C. A. Parker, J. C. Roberts, S. M. Bedair, M. J. Reed, S. X. Liu, N. A. El-Másry, and L. H. Robins, Appl. Phys. Lett. 75, 2566 (1999).

${ }^{28}$ D. Gershoni and H. Temkin, J. Lumin. 44, 381 (1989). 
Applied Physics Letters is copyrighted by the American Institute of Physics (AIP). Redistribution of journal material is subject to the AIP online journal license and/or AIP copyright. For more information, see http:/ojps.aip.org/aplo/aplcr.jsp

Copyright of Applied Physics Letters is the property of American Institute of Physics and its content may not be copied or emailed to multiple sites or posted to a listserv without the copyright holder's express written permission. However, users may print, download, or email articles for individual use. 